\title{
Importance de la protéolyse du lait sur la croissance des bactéries lactiques \\ Utilisation d'une souche de microcoque caséolytique en vue de la maturation et de la transformation du lait en fromagerie de gruyère
}

\author{
par \\ M. FEUILLAT, M. DEMAIMAY, J. C. GAUDIN, P. GRUDE \\ et C. DUMARCHE \\ Laboratoire de chimie et de technologie agricole \\ E.N.I.T.A. de Dijon-Quetigny
}

Depuis quelques années, l'introduction de nouvelles techniques dans la récolte et la conservation du lait avant sa transformation a été la cause de difficultés de fabrication en fromagerie de gruyère.

En particulier, d'après les observations que nous avons pu recueillir, la flore lactique apportée au lait sous la forme de levain se développe difficilement et il en résulte au niveau de la fabrication un certain nombre d'anomalies dans la coagulation, l'égouttage et l'acidification.

L'amélioration des conditions de propreté et d'hygiène dans la récolte du lait est parfois mise en cause. La flore lactique naturelle est moins abondante et moins active et il peut y avoir, par contre, sélection d'une flore constituée en majorité de germes indésirables, voire même dangereux, devenant concurrente des levains apportés ultérieurement.

Un autre motif que l'on peut invoquer et qui nous a en partie orienté vers cette étude est l'abandon progressif de la maturation que l'on faisait subir au lait à $+12^{\circ} \mathrm{C}-+15^{\circ} \mathrm{C}$ avant sa mise en fabrication. Des techniques nouvelles telles que la réfrigération du lait à la ferme, son ramassage en vrac, puis son stockage à l'usine dans des tanks réfrigérés se développent de plus en plus et tendent à supplanter cette pratique.

Peu de données scientifiques existent sur les transformations physico-chimiques du lait au cours de la maturation pratiquée soit d'une façon traditionnelle (maturation en rondots), soit d'une façon plus dirigée (report et thermisation). 
Dans une étude publiée en 1969, Vorob'ev et Ju. Ozola [1] signalent l'influence favorable de la maturation du lait sur la fabrication et l'affinage de fromages de type Hollande. Ces mêmes auteurs constatent un enrichissement en azote soluble et en azote non protéique des fromages élaborés à partir de lait maturé.

Les travaux de Desmazeaud et de Devoyod [2] ont mis en évidence la stimulation des bactéries lactiques thermophiles par les microcoques caséolytiques et la nature peptidique des substances stimulantes. Les préparations commerciales appelées " activateurs " de croissance des levains lactiques telles que celle étudiée par Weber [3] renferment également des peptides.

Ces différentes observations ou études nous ont conduits à rechercher si la maturation du lait était accompagnée d'un phénomène de protéolyse et si l'on pouvait établir une relation entre ce phénomène et la croissance des bactéries lactiques. Dans une deuxième partie de notre étude, nous avons provoqué une protéolyse du lait par ensemencement avec une souche de microcoque caséolytique : la souche M 96 de la station de Recherches laitières de Jouy-en-Josas, et nous avons étudié les conditions de stimulation sur une souche de Streptococcus lactis utilisée dans la préparation des levains en fromagerie de gruyère.

Les résultats obtenus ont fait l'objet d'essais de maturation du lait à l'aide de cette souche de microcoque et certains de ces essais ont été complétés par des fabrications industrielles de fromages à pâte cuite du type Emmental.

A. - Mise en évidence des différences de développement d'un levain lactique mésophile dans trois types de lait : lait frais, lait réfrigéré, lait de maturation traditionnelle, et relation avec le degré de protéolyse de ces laits.

\section{1) Etude de la croissance du levain lactique}

\section{$1^{\circ}$ Protocole de l'essai}

Trois échantillons de laits crus provenant du même mélange sont utilisés pour l'expérience :

- le premier au moment de son arrivée à l'usine : lait frais,

- le second après avoir été refroidi à $4^{\circ} \mathrm{C}$ pendant $12 \mathrm{~h}$ : lait réfrigéré,

- le troisième après avoir été reporté à $15^{\circ} \mathrm{C}$ pendant $12 \mathrm{~h}$ : lait de maturation traditionnelle.

Chacun de ces échantillons est ensemencé à 2 p. 100 par un mélange de souches mésophiles, distribué comme levain en fabrication de Gruyère de Comté par la station I.N.R.A. de Poligny. Les échantillons sont ensuite placés au bain-marie à $30^{\circ} \mathrm{C}$. Des prélèvements sont effectués toutes les $15 \mathrm{mn}$ pendant $1 \mathrm{~h}$, puis soumis à un test à la résazurine avec lecture photométrique selon la technique utilisée par 


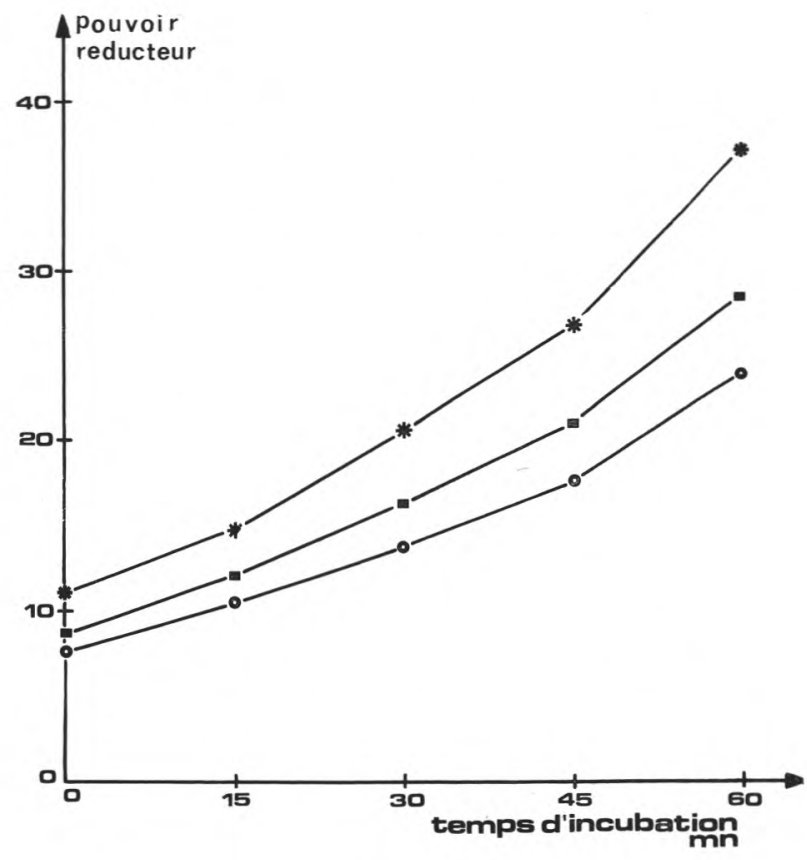

figure 1

Croissance des bactéries lactiques dans trois types de laits

* _ * lait de maturation traditionnelle.

- - lait frais.

- - lait réfrigéré.

Feuillat et al. [4]. Chaque note obtenue est corrigée par rapport à un échantillon témoin de même nature, non ensemencé en ferments lactiques et mis à incuber à $30^{\circ} \mathrm{C}$.

\section{Résultats}

La figure 1 regroupe les courbes obtenues en faisant la moyenne arithmétique de six essais.

Ce graphique traduit bien les différences de croissance du levain lactique dans les trois types de lait.

La multiplication microbienne est nettement stimulée dans le lait dit de maturation traditionnelle $\left(12 \mathrm{~h}\right.$ à $\left.15^{\circ} \mathrm{C}\right)$ alors qu'elle est ralentie dans le lait réfrigéré $\left(12 \mathrm{~h}\right.$ à $\left.4^{\circ} \mathrm{C}\right)$, le comportement dans le lait frais étant intermédiaire. 


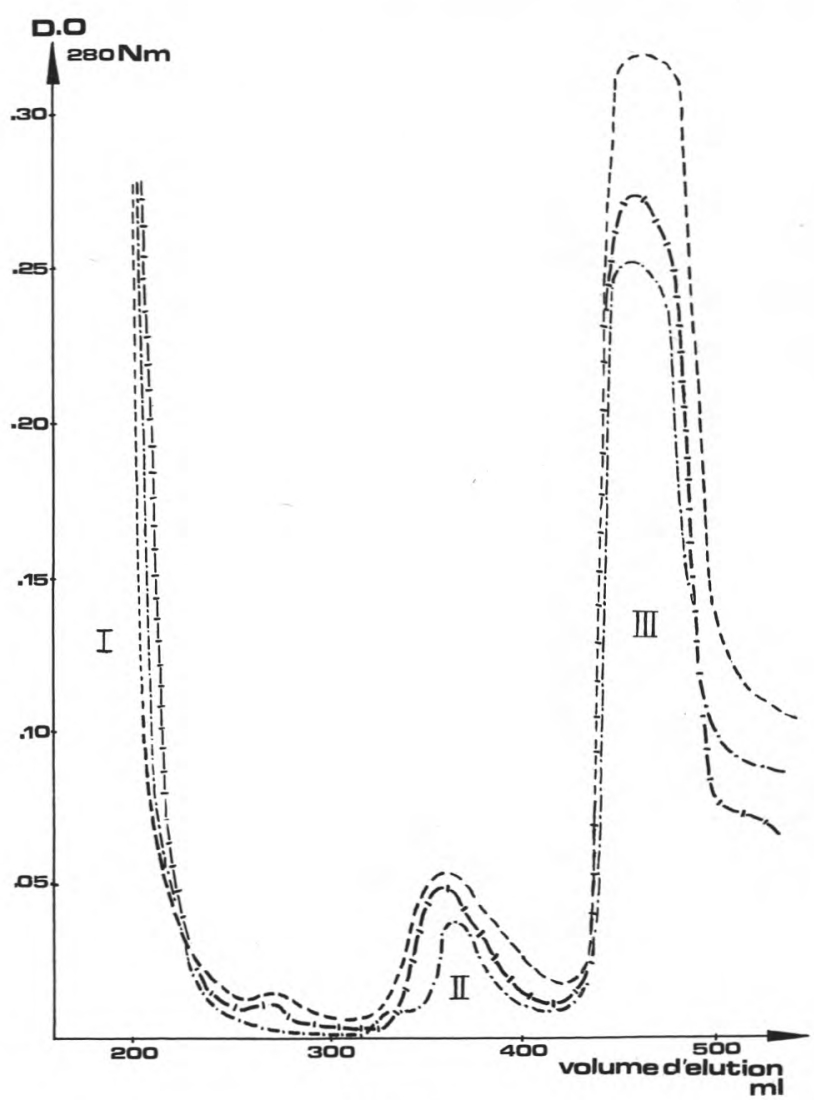

figure 2

Chromatographie sur Séphadex G 25 des fractions azotées solubles de trois types de lait

- - lait de maturation traditionnelle.

-1- lait frais.

-...- lait réfrigéré.

\section{2) Etude du degré de protéolyse des laits}

\section{Technique}

Le but de l'analyse étant la mise en évidence d'une libération de peptides et d'acides aminés dans le lait, c'est-à-dire d'azote soluble, on élimine la caséine par précipitation iso-électrique de trois échantillons de lait de même origine que les précédents mais non ensemencés en ferments lactiques, puis on chromatographie les sérums sur gel de dextrane réticulé (Séphadex G 25). 
Le principe de l'analyse est un tamisage moléculaire.

Du point de vue mode opératoire, le gel est mis à gonfler dans une solution de $\mathrm{NaCl} 0,05 \mathrm{M}$ suivant la technique de Porath [5], puis coulé dans une colonne pharmacia d'une hauteur de $100 \mathrm{~cm}$ et d'un diamètre de $4,5 \mathrm{~cm}$. Après stabilisation du gel par passage d'un volume d'éluant égal à deux fois le volume de la colonne, on chromatographie $10 \mathrm{ml}$ de sérum qui sont élués par une solution d'acide acétique $0,1 \mathrm{M}$ avec un débit de $50 \mathrm{ml} / \mathrm{h}$. On collecte environ $600 \mathrm{ml}$ d'éluat par fractions de $3 \mathrm{ml}$ qui sont ensuite étudiées par absorption dans l'U.V. à $278 \mathrm{~nm}$ à l'aide d'un spectrophotomètre équipé d'un enregistreur.

\section{$2^{\circ}$ Résultats}

La figure 2 représente les trois chromatogrammes obtenus respectivement à partir des sérums de lait frais, de lait réfrigéré et de lait de maturation traditionnelle. Chaque chromatogramme comporte trois pics principaux qui correspondent à trois fractions d'azote organique soluble contenues dans le sérum et séparées par ordre de poids moléculaires décroissants :

- La fraction I (pic tronqué) correspond au volume d'exclusion de la colone $\left(\mathrm{V}_{o}=165 \mathrm{ml}\right)$ et renferme donc les molécules d'un poids supérieur à 5000 , en particulier les protéines solubles.

- La fraction II renferme des peptides qui, étant donné leur zone d'élution (comprise entre 300 et $380 \mathrm{ml}$ ), ont un poids moléculaire pouvant être évalué de 1000 à 1500 si l'on se réfère aux chromatographies effectuées dans des conditions semblables par Desmazeaud et Devoyod.

- La fraction III renferme des peptides très courts et des acides aminés libres, avec en particulier des acides aminés aromatiques étant donné la forte absorption dans l'U.V.

L'examen de ces chromatogrammes indique des différences significatives dans le degré de protéolyse des laits. Ainsi, l'échantillon maturé $12 \mathrm{~h}$ à $15^{\circ} \mathrm{C}$ s'avère le plus dégradé. C'est-à-dire celui dont les fractions peptidiques et acides aminés sont les plus importantes. Ces mêmes fractions semblent avoir disparu en partie dans le lait réfrigéré puisqu'elles sont plus faibles dans celui-ci que dans le lait frais.

\section{3) Conclusion}

Une relation assez étroite semble exister entre le développement d'un levain lactique et le degré de protéolyse du lait ensemencé.

Nous référant de nouveau aux travaux de Desmazeaud et Devoyod qui identifient les peptides d'un poids moléculaire compris entre 1000 et 1500 comme exerçant la plus forte stimulation sur les bactéries lactiques, on peut estimer que la fraction II mise en évidence dans nos propres essais est particulièrement importante, et que l'un 
des rôles de la maturation du lait correspond à un enrichissement en cette fraction de nature peptidique, sous l'action d'une flore protéolytique naturelle. Lorsque le lait est réfrigéré, cette flore ne peut plus se développer ou est concurrencée par une flore psychrophile qui, au contraire, appauvrirait le milieu en peptides et en acides aminés.

Des essais de protéolyse artificielle à l'aide d'une souche de microcoque nous ont donc paru particulièrement intéressants et d'actualité, puisque la maturation traditionnelle du lait est pratiquement abandonnée dans la région de fabrication du gruyère et qu'au contraire la réfrigération à la ferme s'y développe et s'y développera de plus en plus.

$$
*
$$

B. - Protéolyse du lait provoquée par une souche de microcoque et action stimulante sur une souche de Streptococcus lactis.

\section{1) Protocole de l'essai}

$1^{\circ}$ Nature des souches bactériennes

- Microcoques : Nous avons utilisé la souche M96 de microcoques caséolytiques isolés et déjà étudiée par Desmazeaud et Devoyod [2]. Cette souche est conservée par repiquage hebdomadaire sur milieu de Chapman incubé $72 \mathrm{~h}$ à $37^{\circ} \mathrm{C}$, puis maintenu à $4^{\circ} \mathrm{C}$.

- Bactéries lactiques : la souche utilisée est la souche de Streptococcus lactis (collection C.N.R.Z.) conservée par repiqiage sur "Plate count agar » incubé $72 \mathrm{~h}$ à $30^{\circ} \mathrm{C}$, puis maintenu à $4^{\circ} \mathrm{C}$.

$2^{\circ}$ Mesures de l'activité protéolytique des microcoques et de la stimulation de St. lactis

- Milieu de culture : Désirant disposer d'un substrat n'ayant subi aucune dégradation thermique ou microbienne, on utilise du lait prélevé immédiatement après la traite. Celui-ci est écrémé par centrifugation à $8000 \mathrm{t} / \mathrm{mn}$ pendant $20 \mathrm{mn}$, puis par filtration sur verre filtré de porosité $n^{\circ} 4$. Le lait est alors stérilisé par filtration sur un filtre "Millipore " type xx420 4700 d'une capacité de $100 \mathrm{ml}$ et équipé d'une membrane type $\mathrm{HA}$ de $0,45 \mu$. Cette membrane est au préalable stérilisée à l'autoclave à $121^{\circ} \mathrm{C}$ et adaptée à son support qui a été lui-même immergé pendant $20 \mathrm{mn}$ dans l'eau bouillante.

La filtration s'effectue sous une pression d'azote de $12 \mathrm{~kg}$ par $\mathrm{cm}^{2}$ et le filtrat est recueilli dans des récipients stériles.

Des contrôles de la stérilisation sont effectués à chaque essai par incubation d'échantillons à $30^{\circ} \mathrm{C}$, et par ensemencement de milieux gélosés eux-mêmes incubés à $30^{\circ} \mathrm{C}$ pendant $72 \mathrm{~h}$. Ce mode de stérilisation s'est révélé très satisfaisant, aucun développement microbien n'ayant jamais été observé sur les cultures. 


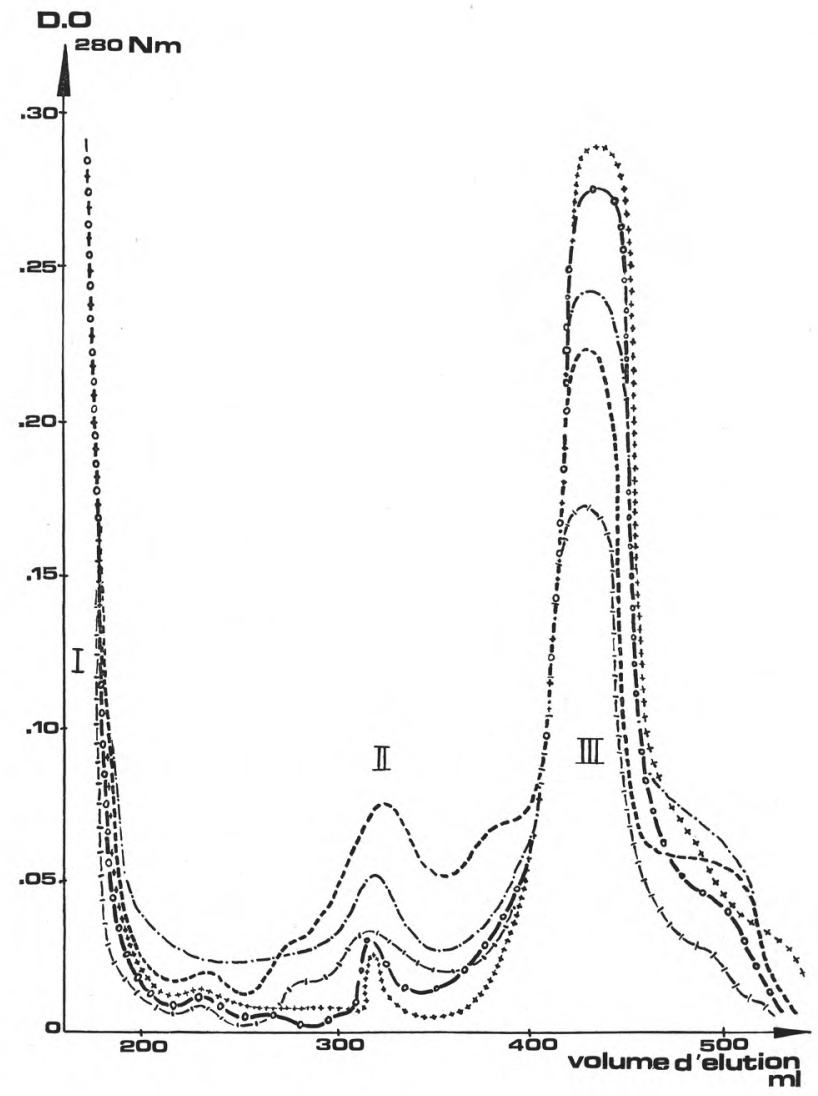

figure 3

Chromatographie sur Séphadex G25 des fractions azotées solubles de laits incubés à $37^{\circ} \mathrm{C}$ avec 1 p. 100 de microcoques :

- $0-0-$ témoin.

$-1-1-\quad$ incubation de $2 \mathrm{~h}$.

- - - incubation de $4 \mathrm{~h}$.

-.-.- incubation de $8 \mathrm{~h}$.

+++ incubation de $12 \mathrm{~h}$. 
- Mesures : huit échantillons de lait stérilisé par filtration sont ensemencés à 1 p. 100 avec un levain de microcoques, puis mis à incuber à $37^{\circ} \mathrm{C}$ :

- deux sont prélevés après $2 \mathrm{~h}$,

- deux après $4 \mathrm{~h}$,

- deux après $8 \mathrm{~h}$,

- deux après $12 \mathrm{~h}$.

Pour chaque groupe d'échantillons :

- l'un est destiné à la mesure de la protéolyse par chromatographie sur Séphadex selon la technique décrite ci-dessus et par microdosage de l'azote dans les différentes fractions selon la technique de Strauch [6] qui comporte une minéralisation et un dosage colorimétrique par le réactif de Nessler,

- l'autre est destiné à la mesure de la stimulation de St. lactis. Pour cela deux techniques sont utilisées : la première consiste à ensemencer l'échantillon à 1 p. 100 par St. lactis dont la croissance est suivie à l'aide du test à la résazurine avec lecture photométrique. La seconde consiste en une culture de St. lactis additionnée seulement de la fraction peptidique libérée dans l'échantillon dégradé par les microcoques.

\section{2) Résultats}

$1^{\circ}$ Activité protéolytique de la souche de microcoques

La figure 3 représente les chromatogrammes obtenus sur Séphadex G 25 pour les différents échantillons.

La fraction II renfermant les substances stimulantes de nature peptidique passe par un maximum pour l'échantillon ayant été mis à incuber pendant $4 \mathrm{~h}$ en présence de microcoques, et décroît ensuite. Pour des incubations de $8 \mathrm{~h}$ et de $12 \mathrm{~h}$, c'est la fraction acides aminés ou fraction III qui augmente aux dépens de la fraction peptidique.

Afin de préciser quantitativement ces phénomènes de protéolyse, nous avons fait un micro-dosage de l'azote dans les différentes fractions.

La figure 4 regroupe les différents résultats obtenus et permet de suivre l'évolution de la teneur en azote des 3 fractions entre $0 \mathrm{~h}$ et $8 \mathrm{~h}$ d'incubation en présence de microcoques.

Il y a confirmation de l'évolution qualitative observée par chromatographie sur Séphadex avec en particulier la mise en évidence d'une libération maximale d'azote peptidique (fraction II) après $4 \mathrm{~h}$ d'incubation. L'azote aminé libre (fraction III) diminue pendant les deux premières heures, ce qui peut correspondre à la multiplication des microcoques, puis augmente d'une façon continue.

L'augmentation permanente de la teneur en composés azotés macro-moléculaires (fraction I) démontre que l'activité protéolytique des microcoques s'exerce pendant toute la durée de l'incubation. 


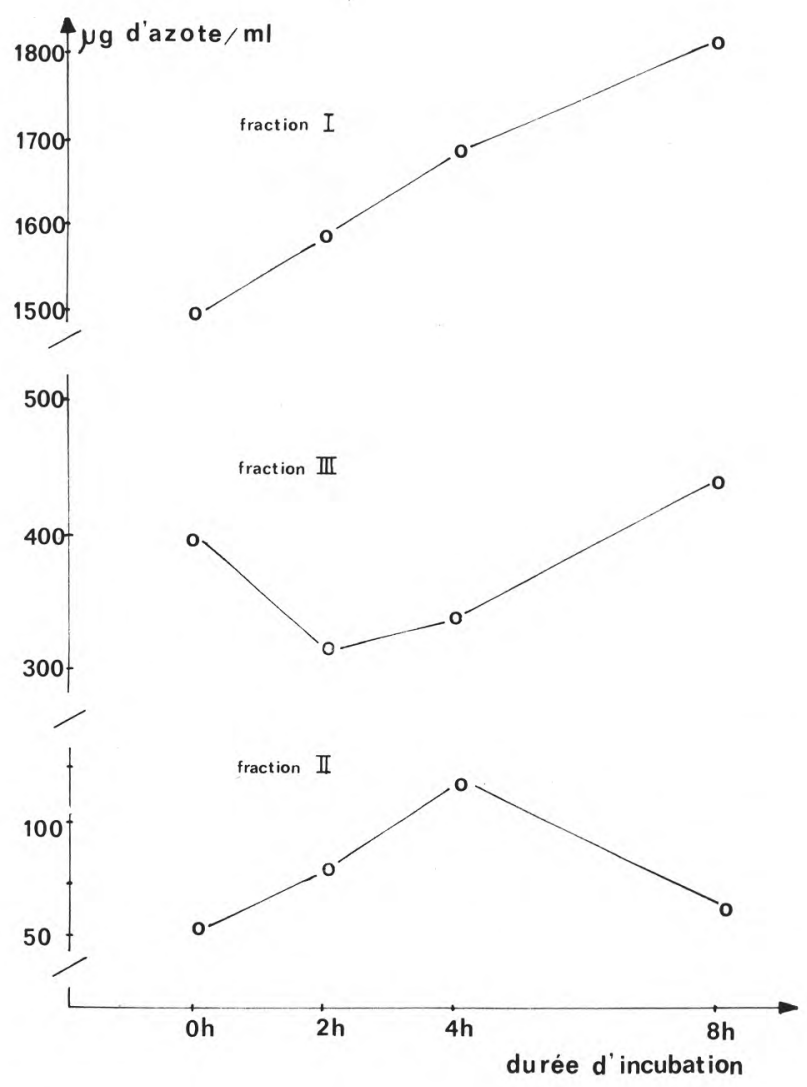

figure 4

Evolution des fractions azotées solubles séparées par chromatographie sur Séphadex G 25 au cours de l'incubation du lait en présence de microcoques.

\section{Action stimulante sur la couche de St. lactis}

Les différences de croissance des bactéries lactiques cultivées sur les différents échantillons de lait dégradé par les microcoques ne peuvent être mises en évidence que par comparaison avec une culture de St. Lactis conduite simultanément sur un lait non dégradé. En effet, étant donné l'intervalle de temps (12 h) qui sépare le premier ensemencement du dernier, il y a inévitablement une évolution du levain lactique utilisé. 

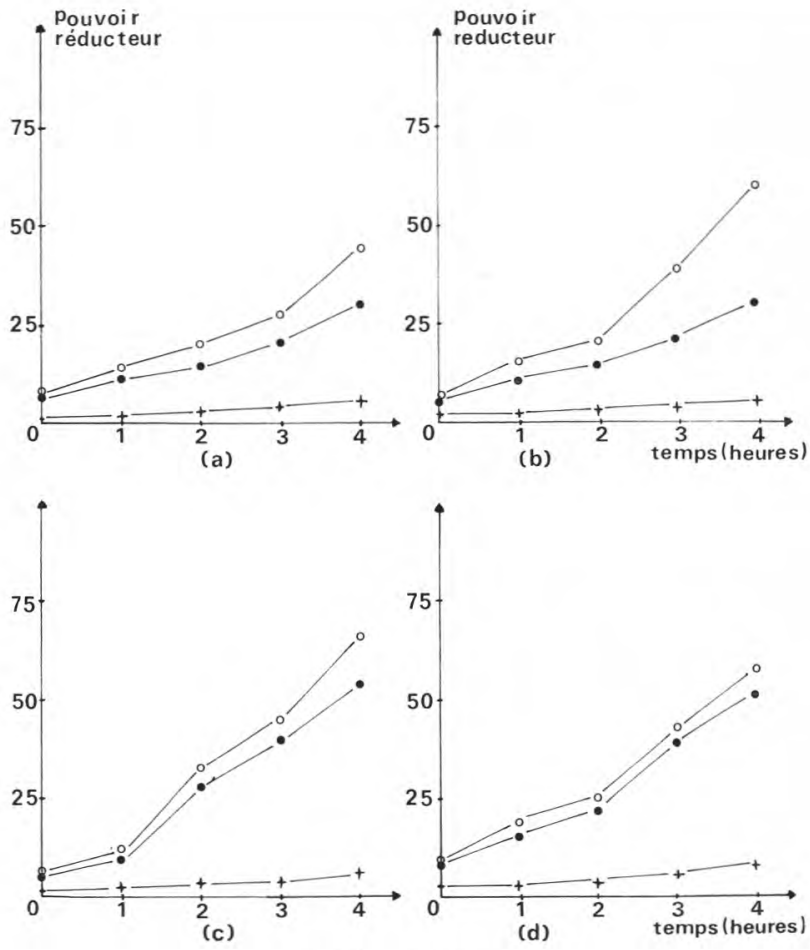

figure 5

Action stimulante des microcoques sur la souche de St. lactis

-O-O- culture sur lait dégradé par les microcoques pendant $2 \mathrm{~h}$ (a), $4 \mathrm{~h}$ (b), $8 \mathrm{~h}$ (c), $12 \mathrm{~h}$ (d).

- - - culture sur lait non dégradé.

$-+-+-+\quad$ culture de microcoques.

Ainsi, la figure 5 regroupe quatre schémas comportant chacun les évolutions simultanées d'une culture de $S t$. lactis sur un lait dégradé par les microcoques, d'une culture de St. lactis sur un lait non dégradé et d'une culture pure de microcoques.

Etant donné, dans tous les cas, le faible pouvoir réducteur de la souche de microcoques, seule, la différence d'activité réductrice entre la culture de St. lactis sur lait dégradé et la culture sur lait non dégradé est bien liée à un phénomène de stimulation. Cette stimulation apparaît avec un maximum d'intensité dans le cas de la culture sur lait préalablement dégradé pendant $4 \mathrm{~h}$ par les microcoques.

Afin d'éliminer les inconvénients dus à l'évolution dans le temps du levain lactique, nous avons effectué des cultures de St. lactis sur du lait frais stérilisé par filtration et additionné seulement des 
fractions d'éluat Séphadex renfermant les peptides (fraction II) libérés par les microcoques.

Ces fractions avaient été préalablement concentrées à $10 \mathrm{ml}$, et en même temps stérilisées, par filtration sur une membrane " Millipore " type P.S.A.C. (membrane pour ultra-filtration à pouvoir de rétention correspondant aux P.M $\geqslant 1000$ ). Elles ont ensuite été ajoutées au lait à la dose de 5 p. 100.

La figure 6 confirme une stimulation maximale de St. lactis par la fraction peptidique provenant d'une dégradation du lait par les microcoques pendant $4 \mathrm{~h}$.

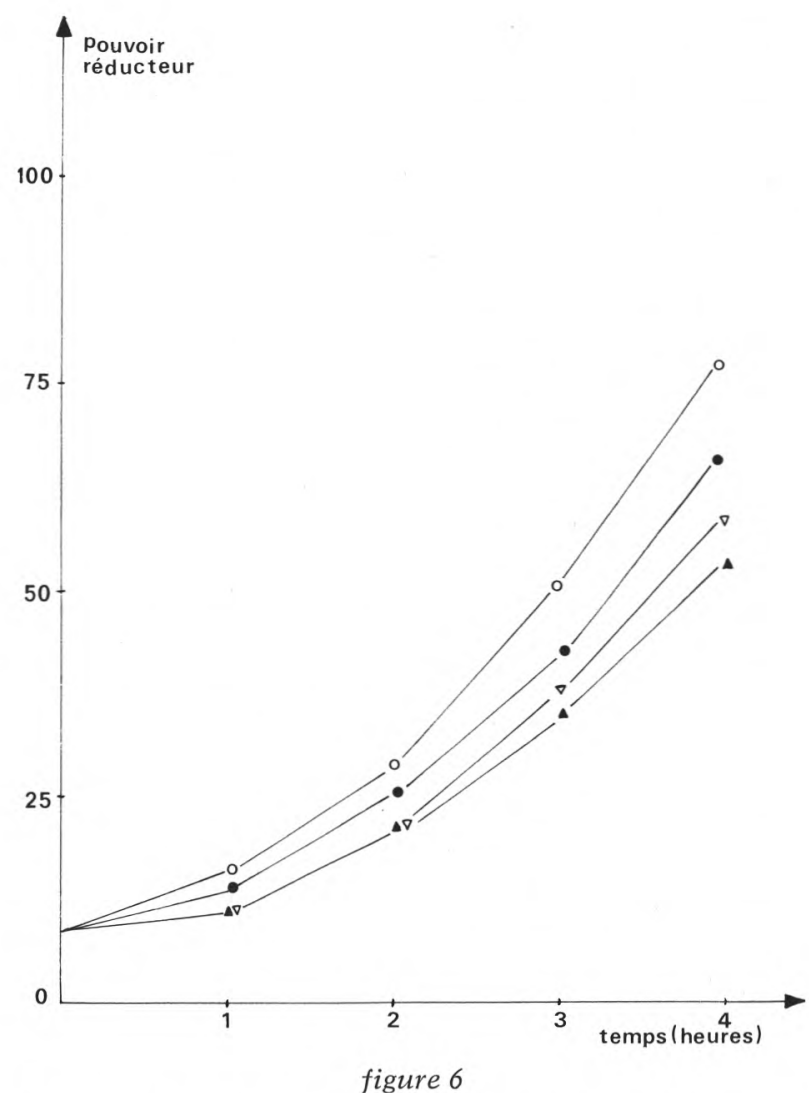

Action stimulante sur St lactis des fractions peptidiques libérées par les microcoques

$\begin{array}{ll}\text { après } 2 \mathrm{~h} \text { d'incubation } & -\triangle-\triangle- \\ \text { après } 4 \mathrm{~h} \text { d'incubation } & -\mathrm{O}-\mathrm{O}- \\ \text { après } 8 \mathrm{~h} \text { d'incubation } & - \\ \text { Témoin } & -\triangle-\triangle-\end{array}$




\section{3) Conclusion}

L'action stimulante des microcoques sur les bactéries lactiques thermophiles mise en évidence par Desmazeaud et Devoyod [2] est vraie également pour des bactéries lactiques mésophiles utilisées dans les levains en fromagerie de gruyère.

Une dégradation du lait de courte durée par les microcoques $(4 \mathrm{~h})$ libère des substances stimulantes de nature peptidique comparables à celles libérées au cours d'une maturation traditionnelle du lait à $15^{\circ} \mathrm{C}$ pendant $12 \mathrm{~h}$.

Ces deux observations nous ont conduit à utiliser la souche de microcoques dans des essais de maturation du lait réalisés à une échelle semi-industrielle et suivis, pour certains, de fabrication de fromages à pâte cuite de type émmental.

Ces essais sont encore actuellement en cours et feront l'objet d'un prochain exposé.

Nous pouvons signaler toutefois que l'expérimentation porte à la fois sur des laits réfrigérés à la ferme et sur des lait non réfrigérés. L'ensemencement et la maturation par les microcoques sont réalisés soit à $30^{\circ} \mathrm{C}$ pendant $4 \mathrm{~h}$ sur une partie seulement du lait à transformer, soit à $16^{\circ} \mathrm{C}$ pendant $12 \mathrm{~h}$ sur la totalité du lait. Les principales observations faites jusqu'à ce jour en fabrication sont un gain d'acidité à l'emprésurage et au pressage. La dose de streptocoques traditionnellement apportés sous la forme de «sérums » doit d'ailleurs être réduite dans d'assez fortes proportions. Des réactions favorables à la présure sont également constatées.

En ce qui concerne la qualité des fromages obtenus, aucune anomalie à l'affinage n'a été constatée. En particulier, il n'y a pas apparition de mauvais goût tel que le goût putride qui pouvait être à redouter dans le cas où il y aurait une protéolyse de la caséine par les microcoques après la fabrication. Au contraire, la plupart des meules fabriquées avec ensemencement par des microcoques ont été notées favorablement quant à l'équilibre de la pâte qui est plus souple que dans les fabrications traditionnelles de même origine.

\section{S u m m a r y}

The important effect of the proteolysis of milk upon the growth of lactic bacteria

Using a strain of caseolytic micrococcus for the maturation and transformation of milk into "Gruyere » cheese

(or Swiss cheese)

A relation is first established between the degree of proteolysis of milk as it is being made and the growth of the mesophyllie lactic 
bacteria provided as leaven. The most proteolysed milks are those which went through a maturation at the $\mathrm{T}^{\circ}$ of $15^{\circ}-16^{\circ} \mathrm{C}\left(\right.$ or $\left.60^{\circ}-62^{\circ} \mathrm{F}\right)$ for 12 hours.

On the contrary, milk chilled on the farm is altered in a very small way.

A short proteolysis of milk with the use of a strain of caseolytic micrococcus releases peptide-like stimulating substances which can be compared with those released during traditional maturation. Now the use of such a strain for the purpose of maturing and transforming milk into Swiss (or Gruyere) cheese is being experimented and put to torial on a semi-industrial scale.

\section{Références bibliographiques}

[1] Vorob'ev (A. I.) et Ozola (Ju.). - Influence d'un lait ayant subi une maturation sur l'affinage et la qualité des fromages. Mémoire original publié dans "Molotchnaja Promychlennost », t. 30, n 4 (avril 1969), p. 11-13, texte Russe traduit en Français par la Technique Laitière.

[2] Desmazeaud (M.) et Devoyod (J. J.) (1970). - Action stimulante des microcoques caséolytiques sur les bactéries lactiques thermophiles. Mise en évidence de la nature peptidique des substances stimulantes. Ann. Biol. Anim. Bioch. Biophys., 10 (3), 412-430.

[3] Weber (F) (1971). - Etude d'un activateur de croissance des levains lactiques. Revue Laitière Française, ${ }^{\circ}$ 286, p. 265-272.

[4] Feuillat (M.), Perrot (Ch.) et Vieudrin (Ch.) (1971). - Essai d'amélioration du test à la résazurine pour le contrôle de la qualité bactériologique du lait. Revue Le Lait, t. LI, n ${ }^{\circ}$ 508, p. 545-558.

[5] PoRATH (J.) (1960). - Gel filtration of proteins, peptides and amino acids. Biochim. Biophys. Acta, 49, p. 193-207.

[6] Strauch (L.) (oct. 1965). - Microméthode pour le dosage de l'azote. Zeitschrift für klinische chemie, fasc. 5 .

\section{Remerciements}

Nous remercions vivement MM. Desmazeaud et Devoyod de la Station Centrale de Recherches Laitières et de Technologie des produits animaux de Jouy-en-Josas de nous avoir procuré la souche de microcoque et de nous avoir communiqué leurs nombreuses observations sur les propriétés de cette souche. 